\title{
THE RAYLEIGH FUNCTION
}

\section{NAND KISHORE}

The present paper is a study of a set of symmetric functions of the zeros of $J_{\nu}(z)$, the Bessel function of the first kind. Let the zeros of $z^{->J_{\nu}}(z)$ be denoted by $j_{\nu, m}, m=1,2, \cdots$, where $\left|R\left(j_{\nu, m}\right)\right|$ $\leqq\left|R\left(j_{\nu, m+1}\right)\right|$. And let

$$
\sigma_{2 n}(\nu) \equiv \sum_{m=1}^{\infty}\left(j_{\nu, m}\right)^{-2 n}, \quad n=1,2, \cdots
$$

We propose to call $\sigma_{2 n}(\nu)$ the Rayleigh function of order $2 n$. The Rayleigh functions of odd orders are identically zero.

These functions were first used by Euler to determine the three smallest zeros of $J_{0}(2 \sqrt{ } z)$; and Rayleigh, independently, calculated the smallest positive zero of $J_{\nu}(z)$ with the aid of these functions. The Rayleigh functions have been the subject of a number of investigations by Cayley, Graeffe, Graf and Gubler, Watson, Kapteyn, Forsyth and others [6, p. 502].

In this paper we shall understand by the Bernoulli and Genocchi numbers, the entities $B_{n}$ and $G_{n}$ defined as follows:

$$
\begin{aligned}
& B_{n}=\sum_{k=0}^{n}\left(\begin{array}{l}
n \\
k
\end{array}\right) B_{k}, n \neq 1, \\
& B_{0}=1 . \quad B_{1}=-\frac{1}{2}, \quad B_{2}=\frac{1}{6}, \quad B_{3}=0, \quad B_{4}=-\frac{1}{30}, \cdots
\end{aligned}
$$

(3) $G_{n}=2\left(1-2^{n}\right) B_{n}$;

(see $[4 ; 3])$.

From the well-known formula

$$
z^{-1 / 2} J_{1 / 2}(z)=\sqrt{\frac{2}{\pi}} \sin z / z,
$$

(see $[6$, p. 54$]$ ), the roots of $z^{-1 / 2} J_{1 / 2}(z)$ are seen to be $k \pi, k=1,2, \cdots$. Hence

$$
\sigma_{2 n}\left(\frac{1}{2}\right)=\sum_{k=1}^{\infty}(k \pi)^{-2 n}=\pi^{-2 n} \zeta(2 n)=(-1)^{n-1} \frac{2^{2 n-1}}{(2 n) !} B_{2 n} .
$$

Similarly, since $z^{1 / 2} J_{-1 / 2}(z)=\sqrt{ } 2 / \pi \cos z$, the roots of $z^{1 / 2} J_{-1 / 2}(z)$ are

Received by the editors December 14, 1961 and, in revised form, May 14, 1962. 
$\left(k-\frac{1}{2}\right) \pi, k=1,2, \cdots$. Therefore,

$$
\sigma_{2 n}\left(-\frac{1}{2}\right)=\pi^{-2 n} \sum_{k=1}^{\infty}\left(k-\frac{1}{2}\right)^{-2 n}=\left(2^{2 n}-1\right) \pi^{-2 n} \zeta(2 n)
$$

$$
=(-1)^{n} \frac{2^{2 n-2}}{(2 n) !} G_{2 n} .
$$

A generating function for $\sigma_{2 n}(\nu)$ can be obtained. Let $J_{\nu}(z)$ be expressed as a Weierstrassian product

$$
J_{\nu}(z)=\frac{\left(\frac{1}{2} z\right)^{\nu}}{\Gamma(\nu+1)} \prod_{n=1}^{\infty}\left\{1-\frac{z^{2}}{j_{\nu, n}^{2}}\right\},
$$

(see [6, p. 498]). Differentiating logarithmically with respect to $z$,

$$
\begin{aligned}
J_{\nu}^{\prime}(z) / J_{\nu}(z) & =\nu / z+\sum_{k=1}^{\infty}(-2 z) /\left(j_{\nu, k}^{2}-z^{2}\right) \\
& =1 / z\left\{\nu-2 \sum_{k=1}^{\infty} \sum_{n=1}^{\infty} z^{2 n} / j_{\nu, k}^{2 n}\right\}, \\
z J_{\nu}^{\prime}(z) / J_{\nu}(z) & =\nu-2 \sum_{n=1}^{\infty} \sigma_{2 n}(\nu) z^{2 n}, \\
\frac{1}{2} z J_{\nu+1}(z) / J_{\nu}(z) & =\sum_{n=1}^{\infty} \sigma_{2 n}(\nu) z^{2 n} .
\end{aligned}
$$

(6)

Substituting $z=1$ in (6) yields

$$
\begin{aligned}
\sum_{n=1}^{\infty} \sigma_{2 n}(\nu) & =\frac{1}{2} J_{\nu+1}(1) / J_{\nu}(1) ; \\
\sum_{n=1}^{\infty} \sigma_{2 n}(\nu+k) & =\frac{1}{2} J_{\nu+k+1}(1) / J_{\nu+k}(1), \quad k=0,1,2, \cdots .
\end{aligned}
$$

Setting $\nu=+\frac{1}{2}$ and $-\frac{1}{2}$ in (6), the following are obtained in view of (4) and (5),

$$
\begin{aligned}
\sum_{n=1}^{\infty}(-1)^{n-1} \frac{2^{2 n}}{(2 n) !} B_{2 n} z^{2 n} & =1-z \cot z \\
\sum_{n=1}^{\infty}(-1)^{n} \frac{2^{2 n-1}}{(2 n) !} G_{2 n} z^{2 n} & =z \tan z
\end{aligned}
$$

Taking the continued fraction representation of the ratio of two 
Bessel functions,

$$
J_{\nu+1}(z) / J_{\nu}(z)=\frac{z}{2(\nu+1)}-\frac{z^{2}}{2(\nu+2)}-\cdots,
$$

(see $[6$, p. 153)] then substituting $z=1$, and using (7),

$$
\sum_{n=1}^{\infty} \sigma_{2 n}(\nu)=\frac{1}{2} \cdot \frac{1}{2(\nu+1)}-\frac{1}{2(\nu+2)}-\cdots .
$$

Set $\nu= \pm \frac{1}{2}$ in (11), then

$$
\begin{aligned}
\sum_{n=1}^{\infty}(-1)^{n-1} \frac{2^{2 n}}{(2 n) !} B_{2 n} & =\frac{1}{3}-\frac{1}{5}-\frac{1}{7}-\ldots \\
& =1-\cot (1)=.3579 \\
\sum_{n=1}^{\infty}(-1)^{n} \frac{2^{2 n-1}}{(2 n) !} G_{2 n} & =\frac{1}{1}-\frac{1}{3}-\frac{1}{5}-\ldots \\
& =\tan (1)=1.5574
\end{aligned}
$$

A recurrence formula for the functions $\sigma_{2 n}(\nu)$ may be derived from (6). Let (6), be written as

$$
\frac{1}{2} z J_{\nu+1}(z)=J_{\nu}(z) \sum_{n=1}^{\infty} \sigma_{2 n}(\nu) z^{2 n}
$$

Substituting the series for $J_{\nu}(z)$ and $J_{\nu+1}(z)$, and identifying the coefficients of $z^{2 n}$ on both sides,

$$
\sum_{k=1}^{n}(-1)^{k-1} 4^{k}(k !)^{2}\left(\begin{array}{c}
n \\
k
\end{array}\right)\left(\begin{array}{c}
\nu+n \\
k
\end{array}\right) \sigma_{2 k}(\nu)=n .
$$

Substitute $\nu= \pm \frac{1}{2}$ in (14), then in view of (4) and (5),

$$
\sum_{k=1}^{n} 2^{2 k-1}\left(\begin{array}{c}
2 n+1 \\
k
\end{array}\right) B_{2 k}=n
$$

(see $[5$, p. 174])

$$
-\sum_{k=1}^{n} 2^{2 k-2}\left(\begin{array}{l}
2 n \\
2 k
\end{array}\right) G_{2 k}=n
$$


which are well-known Bernoulli and Genocchi recurrence formulas.

A determinant representation of $\sigma_{2 n}(\nu)$ may be given. If in (14) the upper limit of the summation is taken as $1,2, \cdots, n$, then $n$ linear equations involving $n$ functions $\sigma_{2}(\nu), \sigma_{4}(\nu), \cdots, \sigma_{2 n}(\nu)$ are obtained. The determinant $\Delta$ of this system is triangular. Hence, the value of $\Delta$ is equal to the product of its elements on the main diagonal,

$$
\Delta=(-1)^{n(n-1) / 2} \cdot 2^{n(n+1)} \cdot n ! ! \prod_{k=1}^{n}(\nu+k)^{n-k+1} .
$$

Then by Cramer's rule,

$$
\sigma_{2 n}(\nu)=(-1)^{n-1} \frac{(n-1) ! !}{n !} 4^{-n} \prod_{k=1}^{n}(\nu+k)^{-n+k-1} \cdot D,
$$

where,

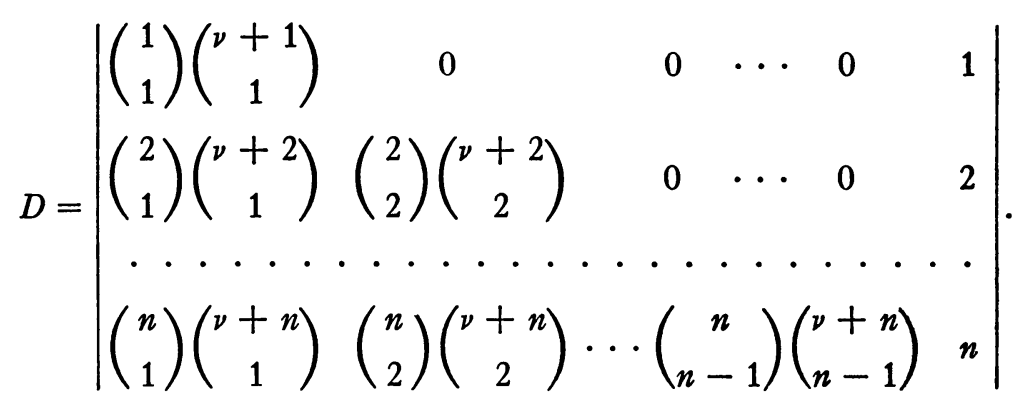

Substitution of $\nu= \pm \frac{1}{2}$ in (17) yields, respectively,

$$
\begin{aligned}
B_{2 n} & =\frac{n !}{(2 n+1) !} 2^{-n+1} D_{1}, \\
G_{2 n} & =-2^{-2 n+2} D_{2},
\end{aligned}
$$

where

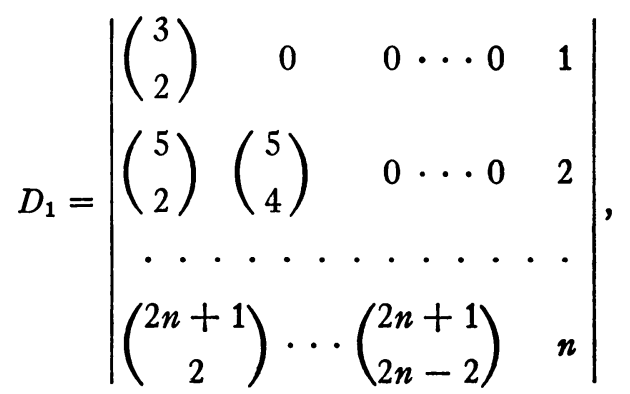




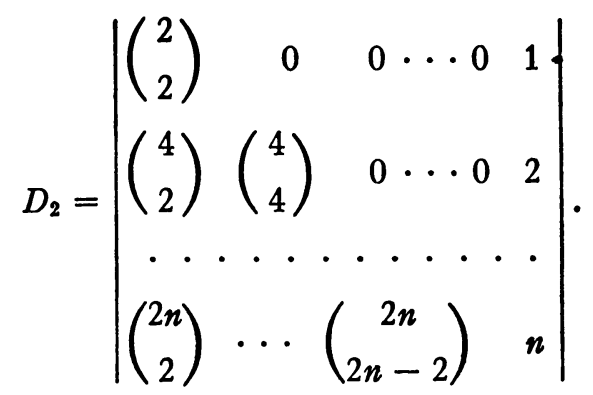

Other formulas for $\sigma_{2 n}(\nu)$ may be derived. Let (6) be written as

$$
\begin{aligned}
\left\{z J_{p+2}(z) / J_{\nu+1}(z)\right\}\left\{z J_{\nu+1}(z) / J_{\nu}(z)\right\} & \\
& =4\left\{\sum_{k=1}^{\infty} \sigma_{2 k}(\nu+1) z^{2 k}\right\}\left\{\sum_{k=1}^{\infty} \sigma_{2 k}(\nu) z^{2 k}\right\} .
\end{aligned}
$$

Then in view of the well-known formula,

$$
J_{\nu+2}(z)=\frac{2(\nu+1)}{z} J_{\nu+1}(z)-J_{\nu}(z)
$$

(see $[6$, p. 45]), the above becomes

$$
-\frac{1}{4} z^{2}+\frac{1}{2}(\nu+1) z J_{p+1}(z) / J_{\nu}(z)=\left\{\sum_{k=1}^{\infty} \sigma_{2 k}(\nu+1) z^{2 k}\right\}\left\{\sum_{k=1}^{\infty} \sigma_{2 k}(\nu) z^{2 k}\right\} .
$$

Identifying the coefficients of $z^{2 n}$ on both sides

$$
(\nu+1) \sigma_{2 n}(\nu)=\sum_{k=1}^{n-1} \sigma_{2 k}(\nu+1) \sigma_{2 n-2 k}(\nu)
$$

Substitution of $\nu=-\frac{1}{2}$, in (20) yields

$$
G_{2 n}=-\sum_{k=1}^{n-1}\left(\begin{array}{l}
2 n \\
2 k
\end{array}\right) B_{2 k} G_{2 n-2 k}
$$

Consider the well-known formulas

$$
\frac{d}{d z}\left(z^{\nu} J_{\nu}(z)\right)=-z^{\nu} J_{\nu+1}(z)
$$

(see $[6$, p. 45]),

$$
\frac{d}{d z}\left(z^{\nu+1} J_{\nu+1}(z)\right)=z^{\nu+1} J_{\nu}(z) .
$$

It follows that 


$$
\frac{d}{d z}\left(\frac{z^{\nu+1} J_{\nu+1}(z)}{z^{-\nu} J_{\nu}(z)}\right)=z^{2 v+1} \frac{\left(1+J_{\nu+1}^{2}(z)\right.}{J_{\nu}^{2}(z)}
$$

That is,

$$
\left(\frac{z}{2} \frac{J_{v+1}(z)}{J_{\nu}(z)}\right)^{2}=-\frac{z^{2}}{4}+\frac{1}{2} z^{-2 v+1} \frac{d}{d z}\left(\frac{z}{2} \frac{J_{\nu+1}(z)}{J_{\nu}(z)} z^{2 \nu}\right) .
$$

Substituting (6) in this relation and identifying the coefficients of $z^{2 n}$,

$$
(\nu+n) \sigma_{2 n}(\nu)=\sum_{k=1}^{n-1} \sigma_{2 k}(\nu) \sigma_{2 n-2 k}(\nu) .
$$

Substitute $\nu= \pm \frac{1}{2}$ in (22), then

$$
-(1+2 n) B_{2 n}=\sum_{k=1}^{n-1}\left(\begin{array}{l}
2 n \\
2 k
\end{array}\right) B_{2 k} B_{2 n-2 k},
$$

(see $[5$, p. 66])

$$
-2(1-2 n) G_{2 n}=\sum_{k=1}^{n-1}\left(\begin{array}{l}
2 n \\
2 k
\end{array}\right) G_{2 k} G_{2 n-2 k} .
$$

Let (6) be multiplied by itself,

$$
J_{\nu+1}^{2}(z)=4 J_{\nu}^{2}(z)\left(\sum_{k=1}^{\infty} \sigma_{2 k}(\nu) z^{2 k-1}\right)^{2},
$$

then substituting the well-known series for $J_{v+1}^{2}(z)$ and $J_{\nu}^{2}(z)$, viz.,

$$
\begin{aligned}
J_{\nu+1}^{2}(z) & =\sum_{k=0}^{\infty}(-1)^{k} \frac{\Gamma(2 \nu+3+2 k)}{k ! \Gamma(2 \nu+3+k) \Gamma^{2}(\nu+2+k)}\left(\frac{z}{2}\right)^{2 \nu+2+2 k}, \\
J_{\nu}^{2}(z) & =\sum_{k=0}^{\infty}(-1)^{k} \frac{\Gamma(2 \nu+1+2 k)}{k ! \Gamma(2 \nu+1+k) \Gamma^{2}(\nu+1+k)}\left(\frac{z}{2}\right)^{2 \nu+2 k},
\end{aligned}
$$

(see $\left[6\right.$, p. 147]), and identifying the coefficients of $z^{2 n}$ on both sides, the following is obtained

$$
\begin{gathered}
\sum_{k=1}^{n}(-1)^{k-1}\left(\begin{array}{c}
2 \nu+2 n-2 k \\
n-k
\end{array}\right)(k !)^{2}\left(\begin{array}{c}
\nu+n \\
k
\end{array}\right)^{2} 4^{k+1} \sum_{s=1}^{k} \sigma_{2 s}(\nu) \sigma_{2(k-s+1)}(\nu) \\
=\left(\begin{array}{c}
2 \nu+2 n \\
n-1
\end{array}\right) .
\end{gathered}
$$

In view of (22) this reduces to 


$$
\begin{gathered}
\sum_{k=1}^{n}(-1)^{k-1} 4^{k}\left(\begin{array}{c}
2 \nu+2 n-2 k \\
n-k
\end{array}\right)\{(k-1) !\}^{2}\left(\begin{array}{c}
\nu+n-1 \\
k-1
\end{array}\right)^{2}(\nu+R) \sigma_{2 k}(\nu) \\
=\frac{2 \nu+1}{2 \nu+n}\left(\begin{array}{c}
2 \nu+2 n-2 \\
n-1
\end{array}\right) .
\end{gathered}
$$

Substitute $\nu= \pm \frac{1}{2}$ in (26), then

$$
\begin{aligned}
& \sum_{k=1}^{n-1}(1+2 k)\left(\begin{array}{c}
2 n \\
2 k
\end{array}\right) B_{2 k}=2 n-1, \\
& \sum_{k=1}^{n-1}(1-2 k)\left(\begin{array}{c}
2 n \\
2 k
\end{array}\right) G_{2 k}=2(2 n-1) G_{2 n} .
\end{aligned}
$$

The Rayleigh functions of odd order are zero,

$$
\sigma_{1}(\nu)=0, \quad \sigma_{3}(\nu)=0, \quad \sigma_{5}(\nu)=0, \cdots .
$$

Identifying the coefficients of $z^{2}$ in (6), an explicit expression for $\sigma_{2}(\nu)$ is obtained. Then (20) may be used to get explicit expressions for the Rayleigh functions of higher orders. Thus,

$$
\begin{aligned}
& \sigma_{2}(\nu)=\frac{1}{2^{2}(\nu+1)} ; \\
& \sigma_{4}(\nu)=\frac{1}{2^{4}(\nu+1)^{2}(\nu+2)} ; \\
& \sigma_{6}(\nu)=\frac{2}{2^{6}(\nu+1)^{3}(\nu+2)(\nu+3)} ; \\
& \sigma_{8}(\nu)=\frac{5 \nu+11}{2^{8}(\nu+1)^{4}(\nu+2)^{2}(\nu+3)(\nu+4)} .
\end{aligned}
$$

The first twelve Rayleigh functions are given in [1, pp. 405-407].

\section{BIBLIOGRAPHY}

1. D. H. Lehmer, Zeros of the Bessel function $J_{\nu}(x)$, Math. Comp. 1 (1943-1945), 405-407.

2. - On the maxima and minima of Bernoulli polynomials, Amer. Math. Monthly 47 (1940), 534-538.

3. - Ann. of Math. (2) 36 (1935), 637-649.

4. E. Lucas, Theorie des nombres.

5. N. Nielsen, Traite elementaire des nombres des Bernoulli.

6. G. N. Watson, $A$ treatise on the theory of Bessel functions, Macmillan, New York, 1944.

UNIVERSITY OF SAN Francisco 